\title{
First detection of a disk free of volatile elements around a young A-type star: A possible sign of collisions between rocky planets ${ }^{\star}$
}

M. E. van den Ancker ${ }^{1}$, N. P. Gentile Fusillo ${ }^{1}$, T. J. Haworth ${ }^{2}$, C. F. Manara ${ }^{1}$, P. A. Miles-Páez ${ }^{1}$, R. D. Oudmaijer ${ }^{3}$, O. Panić ${ }^{3}$, D. J. M. Petit dit de la Roche ${ }^{1}$, M. G. Petr-Gotzens ${ }^{1}$, and M. Vioque $e^{4,5}$

\author{
${ }^{1}$ European Southern Observatory, Karl-Schwarzschild-Str. 2, 85748 Garching bei München, Germany \\ e-mail: mvandena@eso.org \\ 2 Astronomy Unit, School of Physics and Astronomy, Queen Mary University of London, London E1 4NS, UK \\ 3 School of Physics and Astronomy, University of Leeds, Woodhouse Lane, Leeds LS2 9JT, UK \\ 4 Joint ALMA Observatory, Alonso de Córdova 3107, Vitacura 763-0355, Santiago, Chile \\ 5 National Radio Astronomy Observatory, 520 Edgemont Road, Charlottesville, VA 22903, USA
}

Received 13 April 2021 / Accepted 2 July 2021

\begin{abstract}
Aims. We present the first detailed analysis of the astrophysical parameters of the poorly studied Sco-Cen member HD 152384 and its circumstellar environment.

Methods. We analyse newly obtained optical-near-IR X-shooter spectra, as well as archival TESS data, of HD 152384. In addition, we use literature photometric data to construct a detailed spectral energy distribution (SED) of the star.

Results. The photospheric absorption lines in the spectrum of HD 152384 are characteristic of an A0V star, for which we derive a stellar mass of $2.1 \pm 0.1 M_{\odot}$ and a stellar age $>4.5 \mathrm{Myr}$. Superimposed on the photospheric absorption, the optical spectrum also displays double-peaked emission lines of Ca II, Fe I, Mg I, and Si I, typical of circumstellar disks. Notably, all hydrogen and helium lines appear strictly in absorption. A toy model shows that the observed emission line profiles can be reproduced by emission from a compact (radius $<0.3 \mathrm{au}$ ) disk seen at an inclination of $\sim 24^{\circ}$. Further evidence for the presence of circumstellar material comes from the detection of a moderate IR excess in the SED, similar to those found in extreme debris disk systems.

Conclusions. We conclude that HD 152384 is surrounded by a tenuous circumstellar disk that, although rich in refractory elements, is highly depleted of volatile elements. To the best of our knowledge, such a disk is unique among young stars. However, it is reminiscent of the disks seen in some white dwarfs, which have been attributed to the disruption of rocky planets. We suggest that the disk around HD 152384 may have a similar origin and may be due to collisions in a newly formed planetary system.
\end{abstract}

Key words. protoplanetary disks - circumstellar matter - stars: emission-line, Be - stars: pre-main sequence stars: individual: HD 152384

\section{Introduction}

HD 152384 (HIP 82714, $G=7.01$ ) is a poorly studied early-type star located in the constellation of Scorpius (Houk 1982; McDonald et al. 2012). From its parallax and proper motions, Rizzuto et al. (2011) and Galli et al. (2018) identified HD 152384 as a member of the 5-10 Myr Sco-OB2 association, suggesting a young age. Based on an anomaly between the HIPPARCos and Gaia Data Release 2 (DR2) proper motions, Kervella et al. (2019) classified HD 152384 as a suspected binary system. More recently, the multiple nature of HD 152384 was confirmed by El-Badry et al. (2021), who identified the source as Gaia EDR3 6026288713470340608 ( $G=13.58$, separation $77.20^{\prime \prime}$ or $9908 \mathrm{au}$ ) as its companion.

HD 152384 is included in a new sample of 246 Gaia-selected young stars for which we are currently analysing UV-near-IR spectroscopy obtained with the X-shooter instrument on the European Southern Observatory's Very Large Telescope (VLT). Within this sample, and to our knowledge within the entire class

\footnotetext{
* Based on observations collected at the European Southern Observatory (ESO) under ESO programme 0101.C-0902(A).
}

of young stellar objects, HD 152384 shows a unique opticalnear-IR spectrum, on which we report in this Letter.

\section{Observations}

A high-quality spectrum of HD 152384 was obtained with X-shooter - a wide-spectral-range (300-2400 nm) spectrograph on the VLT (Vernet et al. 2011) - on July 2, 2018. Total integration times were $60 \mathrm{~s}, 60 \mathrm{~s}$, and $100 \mathrm{~s}$ for X-shooter's UV, visual, and near-IR arms, respectively. The X-shooter entrance slits were oriented along the parallactic angle $\left(-93.9^{\circ}\right)$ to minimise atmospheric diffraction losses, and slit widths were set to $1.0^{\prime \prime}, 0.9^{\prime \prime}$, and $0.6^{\prime \prime}$ for the UV, visual, and near-IR arms, resulting in spectra with a spectral resolution of 5400,8900, and 8100, respectively. Data were reduced using the X-shooter data reduction pipeline version $3.3 .5^{1}$, after which the spectrum was corrected for telluric absorption using a synthetic transmission spectrum computed using the molecfit package ${ }^{2}$, fitted to the visual and near-IR data. The resulting spectrum is shown in Fig. 1.

\footnotetext{
1 http://www.eso.org/sci/software/pipelines/

2 http://www.eso.org/sci/software/pipelines/skytools/ molecfit
} 


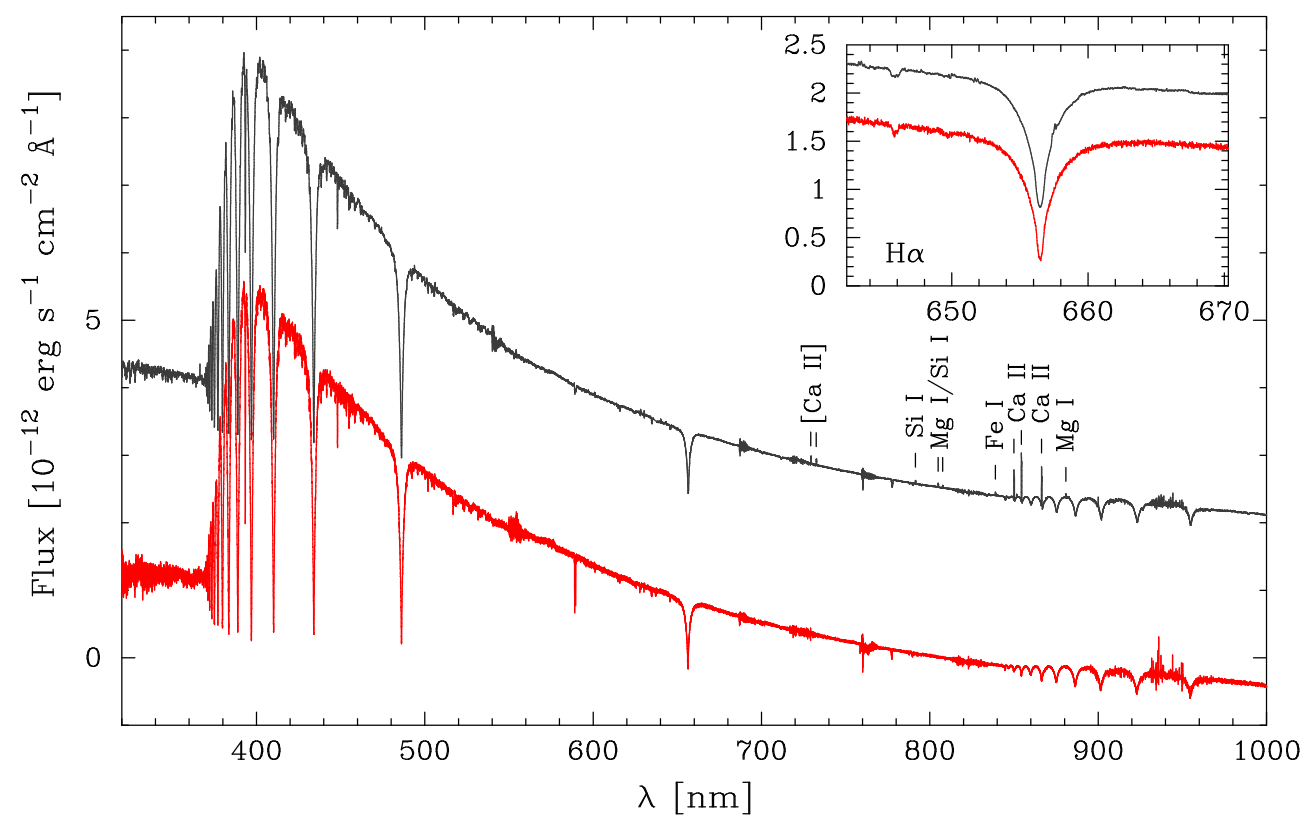

Fig. 1. UV-optical X-shooter spectrum of HD 152384 (black line) with the location of the most prominent emission lines indicated. Also shown (red line) is the spectrum of HD 207222 (spectral type A0), obtained from the X-shooter Spectral Library (Gonneau et al. 2021), shifted for clarity. The inset shows the region around the $\mathrm{H} \alpha$ line $(656.3 \mathrm{~nm})$.

\section{Stellar parameters}

The X-shooter spectrum of HD 152384 (Fig. 1) is dominated by strong absorption lines from H I and the Ca II H $(396.8 \mathrm{~nm})$ and K (393.4 nm) lines. From a Gaussian fit to these absorption lines we derive a heliocentric radial velocity for the star of $-10 \pm 2 \mathrm{~km} \mathrm{~s}^{-1}$. We compared the spectrum of HD 152384 with a template spectrum for an A0 star (HD 207222), taken from the X-shooter Spectral Library (Gonneau et al. 2021). The stellar absorption lines seen in this spectrum are a near-perfect match to the absorption lines seen in HD 152384 (Fig. 1), confirming its previous spectral classification of A0 V (Houk 1982). We also derive a value for the stellar $v \sin i$ of $116 \pm 20 \mathrm{~km} \mathrm{~s}^{-1}$ from the full width half maximum of the $\mathrm{Mg}$ II $448.1 \mathrm{~nm}$ line (seen in absorption) using the method of Royer et al. (2002), correcting for the lower spectral resolution of X-shooter relative to that of the instrument used by Royer et al. (2002).

Using optical photometry retrieved from the SIMBAD database, as well as UV, near-IR, and mid-IR data retrieved from the TD1 (Thompson et al. 1978), 2MASS (Cutri et al. 2003), and AllWISE (Cutri et al. 2012) catalogues, we constructed the spectral energy distribution (SED) of HD 152384 (Fig. 2). As is shown in this figure, the optical and near-IR photometry of HD 152384 can be fitted well by a mildly extincted $\left(A_{V}=0.09\right)$ model for the photosphere of an A0 V star $\left(T_{\text {eff }}=9500 \mathrm{~K}\right)$, whereas a moderate $\left(L_{\mathrm{IR}} / L_{\star}=0.05\right)$ excess emission above the expected photospheric levels can be seen longwards of 2 microns. Using the integrated stellar flux from the fitted Kurucz (1991) model for the stellar photosphere, as well as a distance of $128.3 \pm 0.6 \mathrm{pc}$ derived from the Gaia Early Data Release 3 (EDR3) parallax (Gaia Collaboration et al. 2020), we derive a stellar radius of $1.9 \pm 0.2 R_{\odot}$ and a stellar luminosity of $27.4 \pm 2.1 L_{\odot}$ for HD 152384 . Using the solar abundance pre-main-sequence evolutionary tracks of Bressan et al. (2012), this combination of stellar temperature and luminosity corresponds to a $2.1 \pm 0.1 M_{\odot}$ star located close to the zeroage main sequence, indicating an age of more than $4.5 \times 10^{6}$ years. This lower limit to the stellar age of HD 152384 is

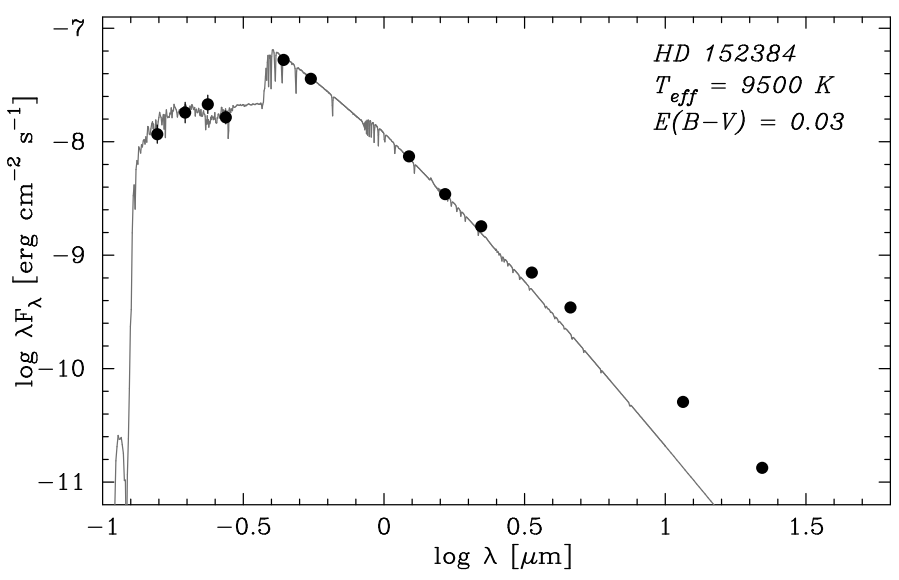

Fig. 2. SED of HD 152384. Observed photometry (black dots) is compared to a Kurucz (1991) model for an A0 $\left(T_{\text {eff }}=9500 \mathrm{~K}\right)$ star (grey line), reddened using the Cardelli et al. (1989) normal extinction law and a colour excess $E(B-V)=0.03$.

compatible with membership in the Sco-OB2 association (e.g. Preibisch \& Mamajek 2008). Our derived stellar parameters are summarised in Table 1.

An inspection of archival TESS (Ricker et al. 2015) data for HD 152384 shows the star to be variable at the $0.1 \%$ level, with a strict periodicity (Fig. 3). We estimated the period by fitting a Gaussian process (GP) with a rotation kernel (Foreman-Mackey et al. 2017, Eq. (56)), which takes the presence of correlated noise in the TESS data into account. We find a period of $8.286 \pm 0.006 \mathrm{~h}$, which we identify with the stellar rotation period. In addition to the regular brightness variations, which we attribute to stellar rotation moving a different pattern of starspots into and out of our line of sight, we also note that a small $(0.2 \%)$ flare is present in the TESS data around 20.5 days, which could be due to further low-level stellar activity. Adopting a stellar radius of $1.9 R_{\odot}$, the rotation period found above corresponds to a rotation velocity of $\sim 280 \mathrm{~km} \mathrm{~s}^{-1}$, or $62 \%$ of 
Table 1. Stellar properties of HD 152384 derived in this paper.

\begin{tabular}{|c|c|c|c|c|c|c|c|c|c|c|}
\hline $\begin{array}{c}d \\
{[\mathrm{pc}]}\end{array}$ & Sp. Type & $\begin{array}{l}T_{\text {eff }} \\
{[\mathrm{K}]}\end{array}$ & $\begin{array}{c}v_{\mathrm{rad}} \\
{\left[\mathrm{km} \mathrm{s}^{-1}\right]}\end{array}$ & $\begin{array}{c}v \sin i \\
{\left[\mathrm{~km} \mathrm{~s}^{-1}\right]}\end{array}$ & $\begin{array}{c}L_{\star} \\
{\left[L_{\odot}\right]}\end{array}$ & $\begin{array}{c}R_{\star} \\
{\left[R_{\odot}\right]}\end{array}$ & $\begin{array}{c}M_{\star} \\
{\left[M_{\odot}\right]}\end{array}$ & $\begin{array}{l}P_{\text {rot }} \\
{[\mathrm{h}]}\end{array}$ & $\begin{array}{c}i \\
{\left[{ }^{\circ}\right]} \\
\end{array}$ & $\begin{array}{c}\text { Age } \\
{[\mathrm{Myr}]}\end{array}$ \\
\hline $128.3 \pm 0.6$ & A $0 \mathrm{~V}$ & $9500 \pm 200$ & $-10 \pm 2$ & $116 \pm 20$ & $27.4 \pm 2.1$ & $1.9 \pm 0.2$ & $2.1 \pm 0.1$ & $8.286 \pm 0.006$ & $24 \pm 4$ & $>4.5$ \\
\hline
\end{tabular}

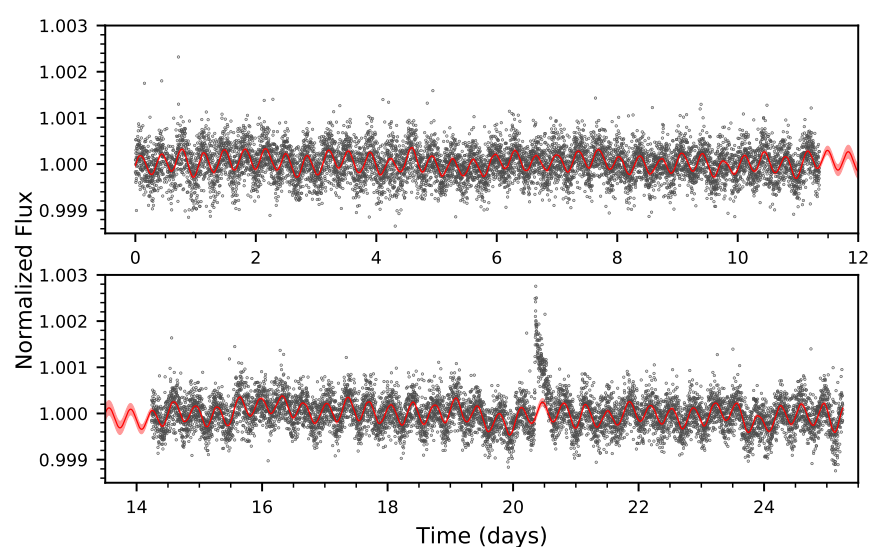

Fig. 3. TESS broadband $(600-1000 \mathrm{~nm})$ normalised photometric time series of HD 152384 (black dots), covering the time period May 21, 2019 , to June 18,2019 . In addition to the periodic variations on an $8.286 \mathrm{~h}$ timescale, a flare can be seen in the data around 20.5 days. The red line shows the GP fitted to the data.

Table 2. Detected emission lines in the spectrum of HD 152384.

\begin{tabular}{|c|c|c|}
\hline$\lambda_{\text {lab. }}[\mathrm{nm}]$ & Ident. & $\mathrm{EW}[\AA]$ \\
\hline 729.15 & [Ca II] & -0.20 \\
\hline 732.39 & [Ca II] & -0.18 \\
\hline 849.81 & $\mathrm{Ca}$ II & -1.60 \\
\hline 854.21 & Ca II & -2.87 \\
\hline 866.21 & Ca II & -2.44 \\
\hline 838.78 & $\mathrm{Fe} I$ & -0.14 \\
\hline 851.41 & $\mathrm{Fe} I$ & -0.19 \\
\hline 882.41 & $\mathrm{Fe} I$ & -0.10 \\
\hline 804.78 & Mg I & -0.15 \\
\hline 880.67 & Mg I & -0.14 \\
\hline 791.38 & $\mathrm{Si} \mathrm{I}$ & -0.12 \\
\hline 807.46 & Si I & -0.09 \\
\hline 835.04 & Si I & -0.05 \\
\hline
\end{tabular}

the stellar break-up velocity of $460 \mathrm{~km} \mathrm{~s}^{-1}$. Although relatively fast for a main-sequence star, this rotation velocity is in line with those found for other young early A-type stars (Wolff et al. 2004; Zorec \& Royer 2012), providing further support for the classification of HD 152384 as a young star. Together with the $v \sin i$ value derived from the $\mathrm{Mg}$ II $448.1 \mathrm{~nm}$ line, we used the rotation velocity to derive a value for the stellar inclination of $i=24 \pm 4^{\circ}$.

\section{Circumstellar material}

There are several indications for the presence of circumstellar material in HD 152384. The IR excess emission seen longwards of $2 \mu \mathrm{m}$ in the SED (Fig. 2) suggests the presence of warm dust in the vicinity of the star. In addition, the X-shooter spectrum displays a number of emission lines superimposed on the

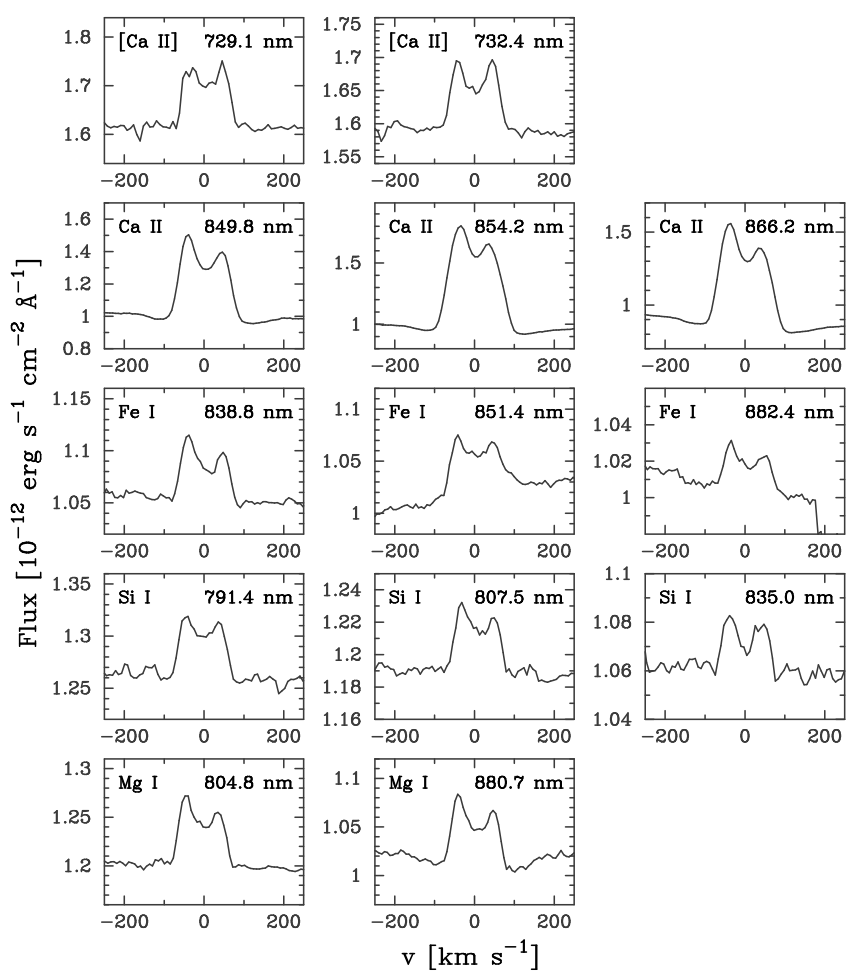

Fig. 4. Detected emission lines in the $\mathrm{X}$-shooter spectrum of HD 152384. Velocity scales for all lines have been corrected for the radial velocity of $-10 \mathrm{~km} \mathrm{~s}^{-1}$ derived from the photospheric absorption lines.

photospheric absorption spectrum. We identified these lines with emission from CaII, Si I, MgI, and Fe I (Table 2). All of the detected emission lines are centred on the stellar radial velocity and thus appear to be clearly associated with HD 152384. The emission lines display a clear double-peaked profile, with a peak-to-peak separation of around $70 \mathrm{~km} \mathrm{~s}^{-1}$ (Fig. 4).

The observed emission line profiles are reminiscent of the line profiles exhibited by the rotating gaseous disks seen around many young stars (e.g., vandenAncker et al. 2004). These lines cannot be due to stellar activity because (1) both the spectral type of A0 V and the low level of variability seen in the TESS data suggest low levels of stellar activity, (2) stellar activity would result in emission components in the $\mathrm{Ca}$ II $\mathrm{H}$ and $\mathrm{K}$ lines (e.g., Vaughan et al. 1978), which are not seen in our data, and (3) stellar activity would not give the double-peaked emission line profiles seen in the $\mathrm{X}$-shooter spectrum. The line profiles are also unlikely to be solely due to a stellar wind as they do not exhibit a $P$ Cygni-like shape. We thus conclude that an origin in a circumstellar disk is the only plausible explanation for the presence of the emission lines seen in the X-shooter spectrum.

With the exception of the [Ca II] lines, which appear to be more symmetric, all detected emission lines display a distinct asymmetry, with the blue peak slightly higher than the red peak. This slight asymmetry could be a reflection of an 
asymmetric distribution of material within the disk or could be due to wind from an optically thick disk (e.g., Ballabio et al. 2020; Pascucci et al. 2020). Alternatively, there could be some additional extinction in our line of sight towards the part of the disk that is moving away from us. Higher spectral resolution data are needed to distinguish between these possibilities.

Following the approach in Carmona et al. (2011), we modelled the detected emission lines using a toy model for gas in a Keplerian orbit around HD 152384, assuming a flat disk with inner radius $R_{\text {in }}$ and outer radius $R_{\text {out }}$. The intensity of the emission decreases as $I(R) \propto\left(R / R_{\text {in }}\right)^{-\alpha}$, where $R$ is the radial distance from the star. The total intensity of the model is computed as a function of velocity for a given inclination, $i$, and convolved with a Gaussian with a width corresponding to the X-shooter spectral resolution in the visual arm. Best fit parameters were selected by comparing a grid of simulated spectra with an average of the continuum-subtracted Ca II emission lines using a $\chi^{2}$ minimisation procedure. Examples of the resulting simulated spectra are shown in Fig. 5.

Assuming that the disk has the same inclination as derived for the stellar photosphere $\left(24 \pm 4^{\circ}\right)$, the emission from the disk needs to originate close to the star $\left(R_{\text {out }}<0.3 \mathrm{au}\right)$ to be able to reproduce the double-peaked emission line profiles seen in the spectra. However, we note that in our model the disk inclination is to some extent degenerate with the disk outer radius, so the emanating region would move correspondingly farther out if the disk were seen under higher inclination than the value derived from the stellar photosphere. The lack of high-velocity wings in the observed line profile may indicate the presence of a small inner gap, which can be reproduced in our model by setting $R_{\text {in }}$ to a value of around $0.03 \mathrm{au}$. Varying $\alpha$, the exponent of the intensity distribution, has only a small effect on the width of the computed line profile. It is therefore poorly constrained by our observations.

What makes the emission-line spectrum of HD 152384 highly unusual is the complete absence of hydrogen and helium emission (cf. Fig. 1), which suggests that the disk is highly depleted in volatile elements. To the best of our knowledge, this spectrum, with strong emission lines from refractory elements but completely lacking emission from volatile species, is unique among young stars. However, similar spectra are seen in rare instances in a completely different class of stars: metal-polluted white dwarfs (Gänsicke et al. 2006; Wilson et al. 2014; Manser et al. 2016; Dennihy et al. 2020; Melis et al. 2020; Gentile Fusillo et al. 2021). Similar to HD 152384, these rare emission line systems also exhibit excess IR emission in their SED that is caused by the presence of circumstellar dust. In the case of these white dwarfs, the dust disk is the result of the tidal disruption of rocky planetesimals (Veras et al. 2015; Malamud \& Perets 2020), and the double-peaked emission lines in their spectrum are thought to originate from a gaseous component in these circumstellar debris disks. The exact mechanism generating the gas is still a matter of debate, and proposed theories include: dust sublimation at the inner edge of the debris disk followed by radial spreading (Metzger et al. 2012), collisional cascades crushing the debris into gas (Kenyon \& Bromley 2017), and the presence of a dense planetesimal orbiting within the disk and disrupting the dust (Manser et al. 2019).

\section{Discussion and conclusions}

HD 152384 appears to be unique among young intermediatemass stars with disks. Its spectrum shows double-peaked $\mathrm{Ca}, \mathrm{Si}$, $\mathrm{Mg}$, and $\mathrm{Fe}$ emission lines but no hydrogen or helium emission,

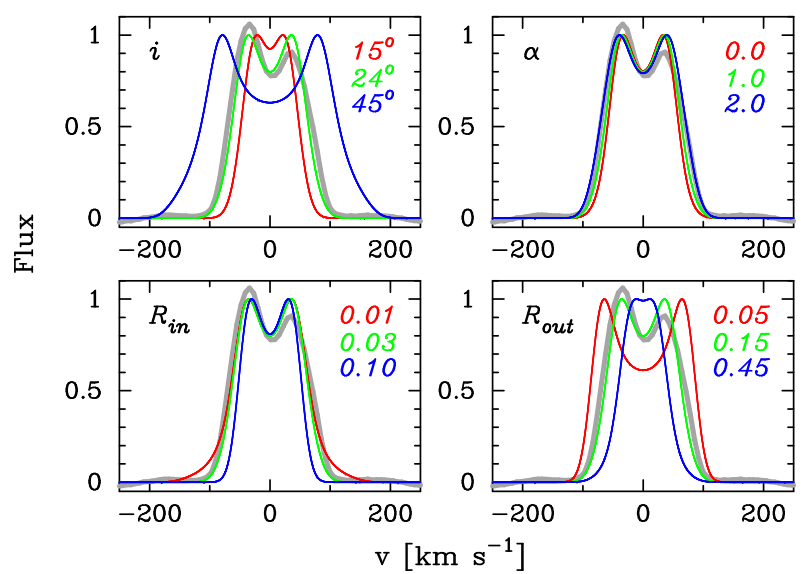

Fig. 5. Simulated emission lines from a flat circumstellar disk using the toy disk model. The grey lines show the continuum-subtracted average Ca II line detected in HD 152384, whereas the green lines show the model with the parameters $i=24^{\circ}$ (corresponding to the stellar inclination), $\alpha=1.0, R_{\text {in }}=0.03$ au, and $R_{\text {out }}=0.15$ au (corresponding to the best fit parameters). In each panel the red and blue lines illustrate the effect of varying one model parameter (top left: inclination; top right: $\alpha$; bottom left: disk inner radius in au; bottom right: disk outer radius in au).

indicative of a disk that, although rich in refractory elements, is highly depleted in volatile materials. The slightly higher blue peak seen in most lines could be due to a slight asymmetry in the disk, could indicate the presence of a disk wind, or could indicate that there is some additional extinction towards the redshifted disk material. Assuming that the star and disk are seen under the same inclination angle of $\sim 24^{\circ}$, the width and peak-to-peak separation of the detected double-peaked emission lines would suggest that these lines originated in a region close (radius $<0.3 \mathrm{au}$ ) to the star.

These properties of HD 152384, although unique for a young star, bear a striking resemblance to a rare subset of metalpolluted white dwarfs, in which the presence of an emission-line spectrum composed solely of refractory elements is commonly explained by the destruction of rocky planets (Bonsor \& Xu 2017). We speculate that similar processes could occur around a young star with a newly formed exoplanetary system, in which the disruption caused by planetary migration could cause collisions between rocky planets. We note that the compact $(<0.3 \mathrm{au})$ disk around HD 152384 is smaller than the disks seen in most Herbig Ae/Be stars (Kraus 2015, and references therein), but its location is similar to that of the rocky planets in our own Solar System. In addition, simulations of tidal disruption of giant planets due to interactions with their host star show that remnants of exomoons can end up in orbits that are similar to the disk seen in HD 152384 (Martinez et al. 2019). Interestingly, its relatively low fractional IR luminosity $\left(L_{\mathrm{IR}} / L_{\star}=0.05\right)$ places HD 152384 within the group of extreme debris disks defined recently by Moór et al. (2021), for which large amounts of warm dust may stem from recent giant impacts between planetary embryos during the final phases of terrestrial planet growth. Although cool molecular gas has been found at relatively large distances in a number of these objects (Moór et al. 2017; Marino et al. 2020), our analysis on HD 152384 constitutes the first detection of gas solely consisting of refractory elements around such an object, leading further support to the hypothesis of the destruction of planetary embryos.

Signatures of selective accretion of circumstellar gas have also been found in the abundance patterns seen in the 
photospheres of $\lambda$ Boo stars and Herbig stars (Venn \& Lambert 1990; Folsom et al. 2012; Kama et al. 2015; Jermyn \& Kama 2018) and may be due to Jupiter-like planets blocking the accretion of part of the dust. Several systems of similar age as HD 152384, such as $\beta$ Pic, also display variable absorption features in their spectrum, which are interpreted as being due to infalling evaporating comets (e.g., Ferlet et al. 1987; Beust et al. 1991; Kiefer et al. 2014; Vidal-Madjar et al. 2017; Rebollido et al. 2020), demonstrating the dynamic nature of newly formed planetary systems. Collisions are also believed to have played an important role in shaping our own Solar System and may have led to the formation of Earth's moon (e.g., Canup \& Asphaug 2001). We postulate that HD 152384 may currently be experiencing a similarly dynamic phase in its evolution. One prediction of our hypothesis of disruption by planetary migration is that HD 152384 should possess one or more giant planets at radii larger than a few tens of au, which would be responsible for the disruption of the inner rocky planets and the creation of the circumstellar disk reported here. High contrast imagers on the next generation of extremely large telescopes will likely be able to detect these.

Acknowledgements. We thank the ESO staff at Paranal and in Garching for their support during the preparation and execution of the observations. We would also like to thank the referee for providing insightful and constructive comments.

\section{References}

Ballabio, G., Alexander, R. D., \& Clarke, C. J. 2020, MNRAS, 496, 2932 Beust, H., Vidal-Madjar, A., \& Ferlet, R. 1991, A\&A, 247, 505

Bonsor, A., \& Xu, S. 2017, ASSL, 445, 229

Bressan, A., Marigo, P., Girardi, L., et al. 2012, MNRAS, 427, 127

Cardelli, J. A., Clayton, G. C., \& Mathis, J. S. 1989, ApJ, 345, 245

Canup, R. M., \& Asphaug, E. 2001, Nature, 412, 708

Carmona, A., van der Plas, G., van den Ancker, M. E., et al. 2011, A\&A, 533, A39

Cutri, R. M., Skrutskie, M. F., van Dyk, S., et al. 2003, The IRSA 2MASS All-Sky Point Source Catalog, NASA/IPAC Infrared Science Archive

Cutri, R. M., Skrutskie, M. F., van Dyk, S., et al. 2012, WISE All-Sky Data Release, NASA/IPAC Infrared Science Archive

Dennihy, E., Xu, S., Lai, S., et al. 2020, ApJ, 905, 5

El-Badry, K., Rix, H.-W., \& Heintz, T. M. 2021, MNRAS, in press [arXiv: 2101.05282]

Ferlet, R., Vidal-Madjar, A., \& Hobbs, L. M. 1987, A\&A, 185, 267

Folsom, C. P., Bagnulo, S., Wade, G. A., et al. 2012, MNRAS, 422, 2072
Foreman-Mackey, D., Agol, E., Ambikasaran, S., \& Angus, R. 2017, AJ, 154, 220

Gaia Collaboration (Brown, A. G. A., et al.) 2020, A\&A, 616, A1

Galli, P. A. B., Joncour, I., \& Moraux, E. 2018, MNRAS, 477, L50

Gänsicke, B. T., Marsh, T. R., Southworth, J., \& Rebassa-Mansergas, A. 2006, Science, 314, 1908

Gentile Fusillo, N. P., Manser, C. J., Gänsicke, B. T., et al. 2021, MNRAS, 504, 2707

Gonneau, A., Lyubenova, M., Lancon, A., et al. 2021, A\&A, 634, A133

Houk, N. 1982, Michigan Spectral Survey (Univ. Michigan Press), 3

Jermyn, A. S., \& Kama, M. 2018, MNRAS, 476, 4418

Kama, M., Folsom, C. P., \& Pinilla, P. 2015, A\&A, 582, L10

Kenyon, S. J., \& Bromley, B. C. 2017, ApJ, 844, 116

Kervella, P., Arenou, F., Mignard, F., \& Thévenin, F. 2019, A\&A, 623, A72

Kiefer, F., Lecavelier des Etangs, A., Boissier, J., et al. 2014, Nature, 514, 462

Kraus, S. 2015, Ap\&SS, 357, 97

Kurucz, R. L. 1991, in Precision photometry: Astrophysics of the galaxy (Schenectady, New York: L. Davis press)

Malamud, U., \& Perets, H. B. 2020, MNRAS, 492, 5561

Martinez, M. A. S., Stone, N. C., \& Metzger, B. D. 2019, MNRAS, 489, 5119

Manser, C. J., Gänsicke, B. T., Marsh, T. R., et al. 2016, MNRAS, 455, 4467

Manser, C. J., Gänsicke, B. T., Eggl, S., et al. 2019, Science, 364, 66

Marino, S., Flock, M., Henning, T., et al. 2020, MNRAS, 492, 4409

McDonald, I., Zijlstra, A. A., \& Boyer, M. L. 2012, MNRAS, 427, 343

Melis, C., Klein, B., Doyle, A. E., Weinberger, A., Zuckerman, B., \& Dufour, P. 2020, ApJ, 905, 56

Metzger, B. D., Rafikov, R. R., \& Bochkarev, K. V. 2012, MNRAS, 423, 505

Moór, A., Curé, M., Kóspál, Á., et al. 2017, ApJ, 849, 123

Moór, A., Ábrahám, P., Szabó, G., et al. 2021, ApJ, 910, 27

Pascucci, I., Banzatti, A., Gorti, U., et al. 2020, ApJ, 903, 78

Preibisch, T., \& Mamajek, E. 2008, Handbook of Star Forming Regions, Volume II, 5, 235

Rebollido, I., Eiroa, C., Montesinos, B., et al. 2020, A\&A, 639, A11

Ricker, G. R., Winn, J. N., Vanderspek, R., et al. 2015, JATIS, 1

Rizzuto, A. C., Ireland, M. J., \& Robertson, J. G. 2011, MNRAS, 416, 3108

Royer, F., Grenier, S., Baylac, M.-O., Gómez, A. E., \& Zorec, J. 2002, A\&A, 393, 897

Thompson, G. I., Nandy, K., \& Jamar, C. 1978, Catalogue of Stellar Ultraviolet Fluxes

Vaughan, A. H., Preston, G. W., \& Wilson, O. C. 1978, PASP, 90, 267

vandenAncker, M. E., Blondel, P. F. C., Tjin A Djie, H. R. E., et al. 2004, MNRAS, 349, 1516

Venn, K. A., \& Lambert, D. L. 1990, ApJ, 363, 234

Veras, D., Eggl, S., \& Gänsicke, B. T. 2015, MNRAS, 451, 2814

Vernet, J., Dekker, H., D’Odorico, S., et al. 2011, A\&A, 536, A105

Vidal-Madjar, A., Kiefer, F., Lecavelier des Etangs, A., et al. 2017, A\&A, 607, A25

Wilson, D. J., Gänsicke, B. T., Koester, D., et al. 2014, MNRAS, 445, 1878

Wolff, S. C., Strom, S. E., \& Hillenbrand, L. A. 2004, ApJ, 601, 979

Zorec, J., \& Royer, F. 2012, A\&A, 537, A120 